\title{
4 Adipositas und Endokrinium
}

\author{
Matthias Blüher
}

Endokrine Erkrankungen wie Hypothyreose, Cushing Syndrom oder Hypogonadismus können in seltenen Fällen zur Entstehung von Adipositas beitragen. In der Praxis ist ein viel häufigeres Problem, dass Adipositas ein unabhängiger Risikofaktor für die Entstehung metabolischer und kardiovaskulärer Erkrankungen ist. Bisher sind die Mechanismen der Adipositasassoziierten Entstehung dieser Folgeerkrankungen nicht vollständig geklärt. Die Adipositasforschung der letzten beiden lahrzehnte hat gezeigt, dass das Fettgewebe ein hochaktives endokrines Organ ist, das eine Vielzahl von bioaktiven Molekülen produziert, die als Adipokine bezeichnet werden. Veränderungen der Konzentration zirkulierender Adipokine wie Leptin, Adiponectin, Interleukin-6 (IL-6), Retinol Bindungsprotein 4 (RBP4), Vaspin, Chemerin, Nampt/PBEF/Visfatin, Adipozyten Fettsäure Bindungsprotein (AFABP), Fibroblasten Wachstumsfaktor 21 (FGF21) und vieler anderer mehr werden häufig bei metabolischen und vaskulären Komplikationen der Adipositas gefunden. Deshalb könnten Veränderungen der Adipokinsekretion das biochemische Bindeglied zwischen vermehrter Fettmasse und gestörter Funktion anderer Gewebe wie Leber und Muskulatur darstellen. Die gestörte Regulation der Adipokinsekretion ist ein wichtiger Mechanismus, über den das Fettgewebe zur Entstehung und Progression von Insulinresistenz und metabolischen Erkrankungen beitragen kann. Auf der anderen Seite ist Adipositas häufig mit endokrinen Veränderungen wie erhöhten Nüchterninsulinspiegeln, einem Hyperkortisolismus und Testosteronmangel beim Mann vergesellschaftet. Zusammengefasst bestehen zwischen Adipositas und endokrinem System enge Wechselwirkungen, die das Fettgewebe selbst als endokrines Organ einschließen.

\subsection{Das Fettgewebe - ein endokrines Organ}

Traditionell wurde das Fettgewebe als ein passives Energiespeicherorgan angesehen. Diese Sicht wurde spätestens in den 8oer-Jahren widerlegt (Kershaw u. Flier 2004). Bereits 1987 wurde nachgewiesen, dass Adipsin, Östrogen und andere Steroidhormone im Fettgewebe nicht nur metabolisiert, sondern auch synthetisiert und sezerniert werden (Kershaw $u$. Flier 2004). Mit der Identifizierung des fettspezifischen Sättigungshormons Leptin etablierte sich das Fettgewebe endgültig als endokrines Organ (Kershaw u. Flier 2004). Heute ist bekannt, dass das Fettgewebe eine Vielzahl bioaktiver Peptide (Adipokine) sezerniert, die sowohl lokale (autokrin/parakrin) als auch systemische (endokrin) Wirkungen haben (s. Abb. 7). Der Begriff „Adipokine“ umfasst alle vom Fettgewebe produzierten und sezernierten Moleküle. Zusätzlich vermehren sich Hinweise, dass das Fettgewebe auch eine Rolle bei der Regulation immunologischer Prozesse spielt.

Zu den Sekretionsprodukten des Fettgewebes gehören unter anderem:

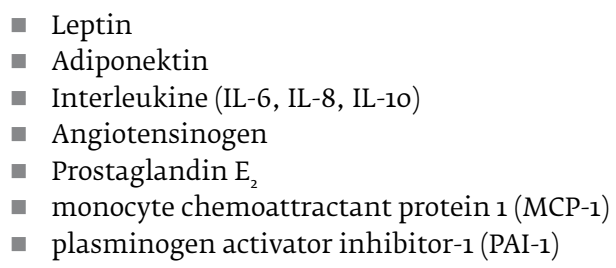




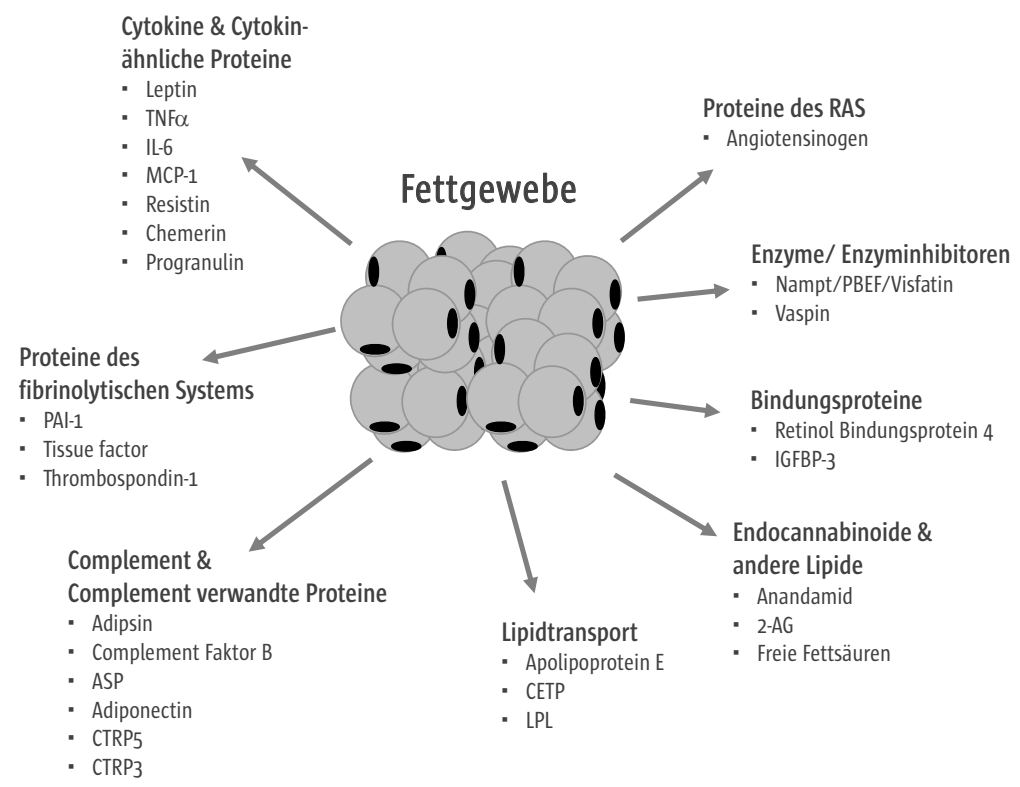

Abb. 7 Das Fettgewebe als endokrines Organ. Beispiele von endokrin wirksamen Molekülen, die aus dem Fettgewebe sezerniert werden

- Resistin

- Retinol Bindungsprotein 4 (RBP4)

- Visfatin

- Vaspin

- Endocannabinoide (Kershaw u. Flier 2004)

Für eine Reihe dieser Adipokine wurden Fettdepotspezifische Unterschiede in der Genexpression, der Produktion oder Sekretion gefunden. Die Suche nach spezifisch aus dem viszeralen Fettgewebe sezernierten Adipokinen ist in den letzten Jahren zum Fokus der Adipositasforschung geworden.

\subsection{Primäre endokrine Erkrankungen und Adipositas}

Obwohl in der Routinediagnostik häufig nach hormonellen Veränderungen bei Übergewicht und Adipositas gesucht wird, finden sich selten primäre endokrine Erkrankungen als Auslöser der Adipositas. Endokrine Erkrankungen, die zur Gewichtszunahme führen sind eine unbehandelte Hypothyreose, Cushing Syndrom, Hypogonadismus, Hypophyseninsuffizienz, Wachstumshormonmangel und andere sehr seltene Erkrankungen (s. Tab. 2). Obwohl ein Hypogonadismus zu Adipositas beitragen kann, ist in der Praxis die Adipositas-assoziierte Suppression des Sexhormone-binding glo- bulin (SHBC), des gesamten und freien Testosterons, beim Mann von größerer klinischer Relevanz. Strukturelle Erkrankungen des Hypothalamus, der eine wesentliche Rolle bei der Regulation von Nahrungsaufnahme und Energieverbrauch spielt, können zu ausgeprägter Adipositas führen. Beispielsweise ist das Prader-Willi-Syndrom als eine genetische Ursache der Adipositas mit einer hypothalamischen Fehlfunktion assoziiert.

\subsection{Auswirkungen der Adipositas auf das Endokrinium}

Die endokrinen Folgen der Adipositas können als physiologische Adaptationen auf eine positive Energiebilanz angesehen werden. Durch das chronische, progrediente Fortschreiten der Adipositas sind diese Anpassungsmechanismen häufig nicht in der Lage, eine Homöostase des entsprechenden Systems zu gewährleisten und werden dann selbst zum Auslöser von Adipositas-Folgeerkrankungen.

\subsubsection{Die hypophysär-adrenale Achse}

Glukokortikoide spielen eine wesentliche Rolle bei der Fettverteilung und Glukokortikoid-Exzess, wie 
Tab. 2 Primäre endokrine Ursachen der Adipositas

\begin{tabular}{lll}
$\begin{array}{l}\text { Strukturelle Erkrankungen } \\
\text { des Hypothalamus }\end{array}$ & Erkrankungen des Endokriniums & $\begin{array}{l}\text { Genetische Adipositasformen mit } \\
\text { Beteiligung des Hypothalamus }\end{array}$ \\
\hline $\begin{array}{l}\text { Kraniopharyngeom } \\
\text { Makroadenome der Hypophyse mit } \\
\text { suprasellärer Ausdehnung }\end{array}$ & Hypothyreose & Prader-Willi-Syndrom \\
\hline $\begin{array}{l}\text { andere Tumoren, Infiltrationen, } \\
\text { Entzündungen des Hypothalamus }\end{array}$ & Wachstumshormonmangel & $\begin{array}{l}\text { Sehr selten: } \\
\text { Leptin- und Leptinrezeptor-Mutationen }\end{array}$ \\
\hline $\begin{array}{l}\text { Trauma, Operationen, } \\
\text { Bestrahlung des Hypothalamus }\end{array}$ & komplette Hypophyseninsuffizienz & PC4 Rezeptor Mutation \\
& M. Cushing & \\
\hline
\end{tabular}

bei Cushing-Syndrom, kann (abdominal-betonte) Adipositas auslösen. Bei Patienten mit Adipositas wurde eine Reihe von Veränderungen der hypophysär-adrenalen Achse beobachtet (Pinkney 2005):

- vermehrte Kortisol-Produktion und-Degradation

- erhöhte Empfindlichkeit gegenüber Stress

- verminderte Kortisol-Tagesrhythmik

- moderat erhöhte ACTH-Spiegel

Die Unterscheidung zwischen Ursache und Folge in der Beziehung zwischen Adipositas und der hypophysär-adrenalen Achse ist allerdings problematisch. Die beiden grundlegenden Veränderungen betreffen eine vermehrte Aktivierung der hypophysär-adrenalen Regulationsmechanismen mit einer verminderten Empfindlichkeit der Glukokortikoid-Rückkopplung und eine erhöhte Empfindlichkeit der Nebenniere und peripherer Organe auf Clukokortikoide (Pinkney 2005).

\subsubsection{Adipositas, Wachstumshormon und Schilddrüsenhormone}

Bei Adipositas kommt es zu einer Reihe von Veränderungen in der Wachstumshormon-IGF1-Achse. Dazu zählen:

- verringerte Wachstumshormon-Sekretion

- erhöhte Produktion des WachstumshormonBindungsproteins

- niedrige Serumspiegel von IGF-1, IGFBP-1 und -3

- erhöhte freie ICF-1 Spiegel in der Zirkulation.

Von diesen Abnormalitäten ist die defiziente Wachstumshormon-Sekretion für die weitere Gewichtszunahme relevant (Pinkney 2005). Schilddrüsenhormone regulieren den Energiestoffwechsel. Bei Adiposi- tas kommt es zu einer erhöhten Aktivität der hypophysär-thyreoidalen Achse, wahrscheinlich infolge eines zentralen Adaptationsmechanismus auf die erhöhte Nahrungsaufnahme. Die Kapazität dieses Anpassungsmechanismus ist allerdings nicht ausreichend, um die Adipositasentwicklung aufhalten zu können.

\subsubsection{Adipositas und Geschlechtshormone}

Geschlechtshormone sind wesentliche Regulatoren der Fettgewebsverteilung und des Stoffwechsels im Fettgewebe. Als Folge der verringerten SHBG-Produktion kommt es einer Erhöhung der freien Geschlechtshormone und deren verstärktem Abbau.

Bei Männern mit Adipositas führt das hauptsächlich zu erniedrigten Testosteronspiegeln, die über eine Verringerung der lipolytischen Aktivität im Fettgewebe zur weiteren Fettakkumulation beitragen können (Pinkney 2005).

Bei Frauen mit Adipositas kommt es hingegen $\mathrm{zu}$ einer erhöhten Androgenproduktion, die für die Entwicklung der zentralen Adipositas prädisponieren. Wahrscheinlich ist Adipositas auch ein kausaler Faktor bei der Entstehung des polyzystischen Ovarialsyndroms, das durch die Kombination aus Insulinresistenz, Hirsutismus und Menstruationsstörungen gekennzeichnet ist. Der pathogenetisch wesentliche Faktor scheint dabei die Adipositas-assoziierte Insulinresistenz zu sein.

\subsubsection{Adipositas, Insulinresistenz und Typ 2 Diabetes}

Viszerale Adipositas wurde in epidemiologischen Studien als unabhängiger Risikofaktor für Typ 2 Diabe- 
tes (Ohlson et al. 1985) identifiziert. Die Diagnose viszerale Adipositas kann nur sicher über bildgebendeVerfahren, wie CT oder MRT, gestellt werden. Für klinisch-praktische Zwecke stellt die Messung des Taillenumfanges in Kombination mit der Messung der subkutanen Hautfaltendicke eine ausreichend gute Methode dar, um Patienten mit hohem Risiko für Adipositas-assoziierte Begleiterkrankungen zu identifizieren.

In der Pathogenese des Typ 2 Diabetes spielt die Kombination aus Insulinresistenz von Muskulatur und Leber, aber auch Fettgewebe, mit einem zunehmenden Insulinsekretionsdefekt der pankreatischen $\beta$-Zellen eine zentrale Rolle. Dabei sind sowohl genetische Faktoren als auch Umwelteinflüsse entscheidend an der Entstehung eines Typ 2 Diabetes beteiligt, wobei die genauen pathogenetischen Mechanismen noch weitgehend unbekannt sind. Zur Beurteilung der Bedeutung des Fettgewebes bei der Ausprägung des Typ 2 Diabetes hat die Generierung gewebespezifischer Insulinrezeptor-Knockout-Mausmodelle wesentlich zum Verständnis der Rolle dieses Organs der Insulinresistenz beigetragen .

Das Fettgewebe spielt eine wichtige Rolle bei der Glukosehomöostase, wie die enge Assoziation zwischen Adipositas, aber auch verschiedenen Syndromen der Lipodystrophie mit Insulinresistenz deutlich machen. Ein wichtiger Mechanismus ist die verminderte Hemmung der Lipolyse durch Insulin. Dadurch werden aus Speichertriglyzeriden vermehrt freie Fettsäuren und Glyzerol freigesetzt, deren Plasmakonzentrationen und Umsatzraten beim Typ 2 Diabetes erhöht sind. Freie Fettsäuren vermindern die muskuläre Glukoseverwertung (Randle-Mechanismus) und stimulieren die hepatische Glukoneogenese sowohl durch Enzymregulation als auch durch Energiebereitstellung. Glyzerol dient dabei als Substrat der beschleunigten Glukoneogenese. Die Insulinresistenz des Fettgewebes beeinflusst also die Glukosehomöostase wahrscheinlich indirekt durch die sekundären Veränderungen in Leber und Muskel.
Für eine kausale Rolle des Fettgewebes bei der Entstehung des Typ 2 Diabetes spricht, dass Mäuse mit einer fettgewebsspezifischen Inaktivierung des insulinabhängigen Clukosetransporters GLUT4 eine Clukoseintoleranz, Hyperinsulinämie und eine Insulinresistenz in Muskulatur und Leber, ähnlich dem Phänotyp des humanen Typ 2 Diabetes, entwickeln (Abel et al. 2001).

\section{Fazit}

Endokrine Erkrankungen wie unbehandelte Hypothyreose, Cushing-Syndrom, Hypophyseninsuffizienz, Wachstumshormonmangel und Hypogonadismus können in seltenen Fällen zur Entstehung von Adipositas beitragen. Im klinischen Alltag sollten die Suche nach einer Schilddrüsenfunktionsstörung und die Bestimmung von Cortisol zur Diagnostik der Adipositas gehören. Auf der anderen Seite kann Adipositas zu endokrinen Veränderungen wie erhöhten Nüchterninsulinspiegeln, einem Hypercortisolismus und Testosteronmangel beim Mann führen. Diese sekundären Veränderungen sind häufig im Rahmen einer gewichtsreduzierenden Therapie reversibel.

\section{Literatur}

Abel ED, Peroni 0, Kim JK, Kim YB, Boss 0, Hadro E, Minnemann T, Shulman GI, Kahn BB (2001) Adipose-selective targeting of the GLUT4 gene impaires insulin action in muscle and liver. $\mathrm{Na}$ ture 409, 729-733

Kershaw EE, Flier JS (2004) Adipose tissue as an endocrine organ. I Clin Endocrinol Metab 89, 2548-2556

Ohlson LO, Larsson B, Svardsudd K, Welin L, Eriksson H, Wilhelmsen L, Björntorp P, Tibblin G (1985) The influence of body fat distribution on the incidence of diabetes mellitus. 13.5 years of follow-up of the participants in the study of men born in 1913. Diabetes 34, 1055-1058

Pinkney I (2005) Obesity and disease: hormones and obesity. In: Clinical Obesity. Kopelmam PG, Caterson ID, Dietz WH (Hrsg.) Blackwell Publishing Ltd., Oxford, UK, $2^{\text {nd }}$ Edition 2005, 198-212

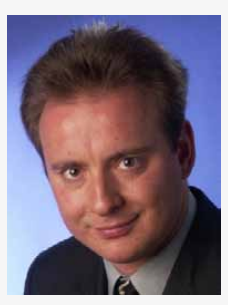

\section{Prof. Dr. med. Matthias Blüher}

Nach Beendigung des Medizinstudiums an der Universität Leipzig begann er seine Facharztausbildung zum Endokrinologen in Leipzig, Zürich und Toronto. Nach einem Forschungsaufenthalt am Joslin Diabetes Center in Boston kehrte er als Leiter einer Nachwuchsgruppe nach Leipzig zurück und wurde 2004 auf eine C3-Professur an die Universität zu Köln berufen. Seit 2006 leitet er die klinische Forschergruppe 152 „Atherobesity“ an der Universität Leipzig. 